Pacific Journal of Mathematics

THE FINITE WEIL-PETERSSON DIAMETER OF RIEMANN 


\title{
THE FINITE WEIL-PETERSSON DIAMETER OF RIEMANN SPACE
}

\author{
SCOTT WOLPERT
}

\begin{abstract}
Let $T_{g}$ be the Teichmüller space and $R_{g}$ the Riemann space of compact Riemann surfaces of genus $g$ with $g \geqq 2$. The space $R_{g}$ can be realized as the quotient of $T_{g}$ by a properly discontinuous group $M_{g}$, the modular group. Various metrics have been defined for $T_{g}$ which are compatible with the standard topology for $T_{g}$ and induce quotient metrics for $R_{g}$. Several authors have considered the Weil-Petersson metric for $T_{g}$. A length estimate derived in a previous paper is summarized; combining this with the Ahlfors Schwarz lemma, an estimate of N. Halpern and L. Keen, and an additional argument shows that the Weil-Petersson quotient metric for $R_{g}$ has finite diameter. A corollary is an estimate relating the Poincaré length of the shortest closed geodesic of a compact Riemann surface to the Poincaré diameter of the surface.
\end{abstract}

For background material the reader is referred to the articles of $\mathrm{L}$. Ahlfors [1] and L. Bers [3] and to the article of L. Bers [5] for a survey of related topics. T. C. Chu $[\mathbf{7 , 8}]$ and $\mathrm{H}$. Masur $[\mathbf{1 2}]$ have obtained results related to ours. The author would like to thank Professor G. Kiremidjian for his assistance.

1. The case of an annulus. Let $A=\{z|1<| z \mid<\rho\}$ be an annulus in the plane. Let $M(A)$ be the space of Beltrami differentials of $A$ endowed with the $L^{\infty}$ metric; let $Q(A)$ be the space of integrable holomorphic quadratic differentials of $A$. An element of $M(A)$ is a tensor of type $(-1,1)$ with measurable coefficient.

Definition 1.1. For $\Phi \in Q(A)$ set

$$
\|\Phi\|_{A}=\left(\int|\Phi|^{2} \lambda_{A}^{-2}\right)^{1 / 2}
$$

where $\lambda_{A}$ is the Poincaré metric of $A$. For $\mu \in M(A)$ set

$$
\|\mu\|_{A}=\sup _{\Phi \in Q(A)}|[\mu, \Phi]| /\|\Phi\|_{A}
$$

where $[\mu, \Phi]=\int_{A} \mu \Phi$. 
The metric $\lambda_{A}$ is known to be given by the following expression

$$
(\pi / \log \rho) \csc (\pi \log |z| / \log \rho)|d z / z| \text {. }
$$

We consider a particular deformation of the annulus

A. For $t \geqq 1$ let $A_{t}=\left\{z_{t}|1<| z_{t} \mid<\rho^{t}\right\}$ then the map

$$
z \mapsto z|z|^{t-1}=z_{t}(z)
$$

is quasiconformal with Beltrami differential

$$
(t-1 / t+1)(z /|z|)^{2} \overline{d z} / d z \text {. }
$$

By considering solutions $\omega(z)$ of the Beltrami differential equation $\omega_{\bar{z}}=\mu \omega_{z}$ where $\mu$ is a Beltrami differential it is seen that the curve of Riemann surfaces $A_{t}$ is represented by the curve

$$
(t-1 / t+1)(z /|z|)^{2} \overline{d z} / d z \subset M(A), \quad t \geqq 1 .
$$

As described in our previous paper [16] $(1 / 2 t)\left(z_{t} /\left|z_{t}\right|\right)^{2} \overline{d z_{t}} / d z_{t}$ is the tangent to this curve at $A_{t}$ expressed as an element of $M\left(A_{t}\right), t \geqq 1$. By Definition 1.1

$$
\begin{aligned}
\|(1 / 2 t) & \left(z_{t} /\left|z_{t}\right|\right)^{2} \overline{d z_{t}} / d z_{t} \|_{A_{t}} \\
= & \sup _{\Phi \in Q\left(A_{t}\right)}\left|\int_{A_{t}}(1 / 2 t)\left(z_{t} /\left|z_{t}\right|\right)^{2} \overline{d z_{t}} / d z_{t} \Phi\right| /\left(\int_{A_{t}}|\Phi|^{2} \lambda_{A_{t}}^{-2}\right)^{1 / 2} .
\end{aligned}
$$

It is clear that the extremal $\Phi$ is given by $\left(d z_{t} / z_{t}\right)^{2}$. The value of the quotient in (1.2) is now equal to

$$
\left(2 \pi^{3} / t^{3} \log \rho\right)^{1 / 2} .
$$

Thus the length of the curve $A_{t}, t \geqq 1$ is given by the convergent integral

$$
\int_{1}^{\infty}\left(2 \pi^{3} / t^{3} \log \rho\right)^{1 / 2} d t
$$

For a compact Riemann surface $R$ of genus $g, g \geqq 2$ one can identify the cotangent space at the point $R$ of Teichmüller space with the regular quadratic differentials $Q(R)$ of $R$ and the tangent space at $R$ with the Beltrami differentials $M(R)$ modulo those which are infinitesimally trivial, [1]. In this instance the Weil-Petersson metric and cometric are given by Definition 1.1 on replacing $A$ by $R,[15]$. 
2. Finite diameter of Riemann space. The Riemann space $R_{g}$ of genus $g, g \geqq 2$ is the space of conformal equivalence classes of similarly oriented compact Riemann surfaces of genus $g,[14]$. A natural projection $\pi_{g}$ of $T_{g}$ to $R_{g}$ exists; this projection can be given by the action of a properly discontinuous group $M_{g}$, the modular group, [6]. S. Kravetz showed that every metric $d($,$) for T_{g}$ compatible with the topology of $T_{g}$ induces a quotient metric $\tilde{d}($,$) for R_{g}$ defined as

$$
\tilde{d}(\tilde{x}, \tilde{y})=\inf _{\substack{\pi_{g}(x)=\tilde{x} \\ \pi_{g}(y)=\tilde{y}}} d(x, y)
$$

for $x, y \in T_{g}$ and $\tilde{x}, \tilde{y} \in R_{g},[\mathbf{1 1}]$.

Definition 2.1. For $\tilde{x}, \tilde{y} \in R_{\mathrm{g}}$ let

$$
\omega(\tilde{x}, \tilde{y})=\inf _{\substack{\pi_{g}(x)=\tilde{x} \\ \pi_{g}(y)=\tilde{y}}} d_{w-p}(x, y)
$$

where $d_{w-p}($,$) is the Weil-Petersson metric for T_{g}$.

Let $H=\{z \mid \operatorname{Im} z>0\}$ denote the upper half plane and $\Delta=$ $\partial^{2} / \partial x^{2}+\partial^{2} / \partial y^{2}$ the Laplacian. The following definition and theorem are due to L. Ahlfors, [2].

Definition 2.2. A metric $\rho|d z|, \rho \geqq 0$ is said to be ultrahyperbolic in $H$ if it has the following properties:

(i) $\rho$ is upper semicontinuous;

(ii) at every $z_{0} \in H$ with $\rho\left(z_{0}\right)>0$ there exists a $\rho_{0}$ defined and of class $C^{2}$ in a neighborhood $V$ of $z_{0}$ such that $\Delta \log \rho_{0} \geqq \rho_{0}^{2}$ and $\rho \geqq \rho_{0}$ in $V$ while $\rho\left(z_{0}\right)=\rho_{0}\left(z_{0}\right)$.

The Poincaré metric of $H$ is $|d z| / y$.

THEOREM 2.3. Let $\rho|d z|$ be an ultrahyperbolic metric for $H$. Then $\rho|d z| \leqq|d z| / y$.

The following theorem is due to L. Bers, [4] and D. Mumford, [13].

THEOREM 2.4. For $c>0$, let $K_{c} \subset R_{g}, g \geqq 2$ consist of those Riemann surfaces $R$ for which each closed Poincaré geodesic has length at least $c$. Then $K_{c}$ is a compact set.

THEOREM 2.5. $R_{g}$ has finite diameter for the $\omega($,$) metric.$

Proof. Consider the following regions in $H C\left(l, \theta_{0}\right)=\{z \mid \operatorname{Im} z>$ 
$\left.0,1<|z|<\exp l, \quad \theta_{0}<\arg z<\pi-\theta_{0}\right\} \quad$ and $\quad \theta_{1}<\theta_{2} \quad C\left(l, \theta_{1}, \theta_{2}\right)=$ $C\left(l, \theta_{1}\right)-C\left(l, \theta_{2}\right)$. The Poincaré area of $C\left(l, \theta_{0}\right)$ (resp. $\left.C\left(l, \theta_{1}, \theta_{2}\right)\right)$ is $2 l \cot \theta_{0}\left(\operatorname{resp} .2 l\left(\cot \theta_{1}-\cot \theta_{2}\right)\right)$. The self map of $H z \mapsto z \exp l$ identifies the boundaries of $C\left(l, \theta_{0}\right)$ such that the quotient $A\left(l, \theta_{0}\right)=$ $C\left(l, \theta_{0}\right) /\{z \mapsto z \exp l\}$ is conformally an annulus. Let $\tilde{C}\left(l, \theta_{1}, \theta_{2}\right)$ denote $C\left(l, \theta_{1}, \theta_{2}\right)$ with the boundaries $\bar{C}\left(l, \theta_{1}, \theta_{2}\right) \cap\left\{z \mid \arg z=\theta_{2}\right\}$ and $\bar{C}\left(l, \theta_{1}, \theta_{2}\right) \cap\left\{z \mid \arg z=\pi-\theta_{2}\right\}$ identified by the map $z \mapsto z \exp i(\pi-$ $\left.2 \theta_{2}\right)$; the quotient $A\left(l, \theta_{1}, \theta_{2}\right)=\tilde{C}\left(l, \theta_{1}, \theta_{2}\right) /\{z \mapsto z \exp l\}$ is conformally an annulus. Let $\alpha(\theta)$ (resp. $\beta(\theta)$ ) denote the projection to $A\left(l, \theta_{0}\right)$ (resp. $\left.A\left(l, \theta_{1}, \theta_{2}\right)\right)$ of the curve $z=r \exp i \theta, 1 \leqq r \leqq \exp l$ provided $\theta_{0} \leqq \theta \leqq$ $\pi-\theta_{0}$ (resp. $\theta_{1} \leqq \theta \leqq \theta_{2}$ ). A quotient metric for $A\left(l, \theta_{0}\right)$ (resp. $\left.A\left(l, \theta_{1}, \theta_{2}\right)\right)$ is obtained from the restriction to $C\left(l, \theta_{0}\right)\left(\operatorname{resp} . C\left(l, \theta_{1}, \theta_{2}\right)\right)$ of the line element $|d z| / y$. The distance between the boundaries of $A\left(l, \theta_{0}\right)$ (resp. $\left.A\left(l, \theta_{1}, \theta_{2}\right)\right)$ in the quotient metric will be referred to as the width of $A\left(l, \theta_{0}\right)$ (resp. $A\left(l, \theta_{1}, \theta_{2}\right)$ ). Since each curve $z=r \exp i \theta \subset H$ $0<\theta<\pi$ is a Poincare geodesic it follows that the width of $A\left(l, \theta_{0}\right)$ is given by the integral $\int_{\theta_{0}}^{\pi-\theta_{0}} r d \theta / r \sin \theta=2 \ln \left(\cot \theta_{0}+\csc \theta_{0}\right)$. The induced quotient metric for $A\left(l, \theta_{1}, \theta_{2}\right)$ is not differentiable on the curve $\beta\left(\theta_{2}\right)$; nevertheless, it is straightforward that the width of $A\left(l, \theta_{1}, \theta_{2}\right)$ is $\left.2 \ln (\cot \theta+\csc \theta)\right|_{\theta_{2}} ^{\theta_{1}}$. The curve $\beta\left(\theta_{2}\right)$ has length $\int_{1}^{\exp l} d r / r \sin \theta_{2}=$ $l \csc \theta_{2}$.

The following lemmas of N. Halpern [9] and L. Keen [10] are essential to our argument.

Lemma 2.6. Let $R$ be a compact Riemann surface. For every $c_{1}>0$ there exists a $c_{2}>0$ such that for $\gamma$ a simple closed Poincaré geodesic of length $l$ at most $c_{1}$, the region $A\left(l, \theta_{l}\right), \theta_{l}=\cot ^{-1}\left(c_{2} / 2 l\right)$, can be isometrically imbedded into $R$ with $\alpha(\pi / 2)$ realizing $\gamma$.

Observe that $2 l \cot \theta_{l}$ represents the area of $A\left(l, \theta_{t}\right)$.

LEMMA 2.7. Let $R$ be a compact Riemann surface of genus $g$, $g \geqq 2$. There exists a constant $c_{3}>0$ such that there are at most $3 g-3$ simple closed Poincaré geodesics of length at most $c_{3}$.

Proof of Lemma 2.7. By Lemma 2.6 one can choose $c_{3}<c_{1}$ such that the width of $A\left(l, \theta_{1}\right)$ for $l \leqq c_{3}$ is at least $c_{3}$. The conclusion now follows since there are at most $3 g-3$ mutually disjoint, homotopically nontrivial, simple closed curves on $R$ which are mutually not freely homotopic.

Let $\Phi_{l}=\cot ^{-1}\left(c_{2} / 4 l\right)$ and consider the domain $A\left(l, \theta_{l}, \Phi_{l}\right)$. The width of $A\left(l, \theta_{l}, \Phi_{l}\right)$ is $\left.2 \ln (\cot \theta+\csc \theta)\right|_{\phi_{l}} ^{\theta_{l}}$ which is bounded from below for $l \leqq c_{3}$ provided there exists a constant $c>0$ such that 


$$
\left(\cot \theta_{l}+\csc \theta_{l}\right) /\left(\cot \Phi_{l}+\csc \Phi_{l}\right) \geqq c \quad \text { for } l \leqq c_{3} .
$$

For $c_{3}$ sufficiently small $\csc \Phi_{l} \leqq 2 \cot \Phi_{l}$ thus

$$
\left(\cot \theta_{l}+\csc \theta_{l}\right) /\left(\cot \Phi_{l}+\csc \Phi_{l}\right) \geqq \cot \theta_{l} / 3 \cot \Phi_{l} \geqq 2 / 3
$$

The length of $\beta\left(\Phi_{l}\right)$ is

$$
l \csc \left(\cot ^{-1}\left(c_{2} / 4 l\right)\right) \geqq l \cot \left(\cot ^{-1}\left(c_{2} / 4 l\right)\right)=c_{2} / 4 .
$$

For an annulus $A=\{z|1<| z \mid<r\}$ we make the following definition.

Definition 2.8. The extremal length $E(A)$ of $A$ is given by $E(A)=2 \pi / \log r$.

Now the extremal length of $A\left(l, \theta_{l}, \Phi_{l}\right)$ is $E\left(A\left(l, \theta_{l}, \Phi_{l}\right)\right)=$ $l / 2\left(\Phi_{l}-\theta_{l}\right)=l / 2\left(\cot ^{-1}\left(c_{2} / 4 l\right)-\cot ^{-1}\left(c_{2} / 2 l\right)\right)$ where by l'Hopital's rule

$$
\lim _{l \rightarrow 0} l / 2\left(\cot ^{-1}\left(c_{2} / 4 l\right)-\cot ^{-1}\left(c_{2} / 2 l\right)\right)=c_{2} / 4
$$

It is now clear that $c^{\prime}, 0<c^{\prime}<c_{3}$ can be chosen such that for $l \leqq c^{\prime}$

$$
\begin{gathered}
\left.2 \ln (\cot \theta+\csc \theta)\right|_{\Phi_{l}} ^{\theta_{l}} \geqq c^{\prime} \\
l \csc \Phi_{l} \geqq c^{\prime}
\end{gathered}
$$

and

$$
l / 2\left(\Phi_{l}-\theta_{l}\right) \leqq c_{2}
$$

These inequalities will now be used to estimate the diameter of $R_{g}$. The region $K_{c^{\prime}} \subset R_{g}$ is compact and thus has finite $\omega$ diameter. Let a Riemann surface $R$ represent a point in $T_{g}$ such that $\pi_{g}(R) \notin K_{c^{\prime}}$ with $\gamma_{1}, \cdots, \gamma_{n}$ the geodesics of $R$ of length less than $c^{\prime}$. The object is to "fatten" $R$ in a neighborhood of each of $\gamma_{1}, \cdots, \gamma_{n}$ thereby obtaining a surface in $K_{c^{\prime}}$. By Lemma 2.6 a region $A\left(l, \theta_{l}\right)$ can be considered as a coordinate neighborhood of $\gamma_{1}$ where $l$ is the length of $\gamma_{1}$. A new surface $R^{*}$ can be formed by removing the part of $A\left(l, \theta_{l}\right)$ corresponding to $A\left(l, \Phi_{l}\right)$ and identifying the boundaries by the map $z \mapsto z \exp i\left(\pi-2 \Phi_{l}\right)$. Thus $A\left(l, \theta_{l}, \Phi_{l}\right)$ represents a coordinate patch in a neighborhood of the gluing and the original coordinates are chosen otherwise. In a neighborhood of the gluing $\left.\lambda_{R}\right|_{R^{*}}$, the Poincaré metric of $R$ restricted to $R^{*}$, is defined in terms of the coordinate patch $A\left(l, \theta_{l}, \Phi_{l}\right)$; for coordinate patches disjoint from the gluing $\left.\lambda_{R}\right|_{R^{*}}=\lambda_{R}$. Assuming that $\left.\lambda_{R}\right|_{R^{*}}$ is 
ultrahyperbolic Theorem 2.3 implies that $\left.\lambda_{R}\right|_{R^{*}} \leqq \lambda_{R^{*}}$ where $\lambda_{R^{*}}$ is the Poincare metric of $R^{*}$. To show that $\left.\lambda_{R}\right|_{R^{*}}$ is ultrahyperbolic it suffices to consider the metric in a neighborhood of the gluing. Define the metric $\tilde{\lambda}(z)|d z|$ on $\tilde{C}\left(l, \theta_{l}, \Phi_{l}\right)$ by setting $\tilde{\lambda}(z)|d z|=|d z| / \operatorname{Im} z$ for $1<|z|<$ $\exp l, \theta_{l}<\arg z<\Phi_{l}$ and $\tilde{\lambda}(z)|d z|=|d z| / \operatorname{Im}\left(z \exp i\left(2 \Phi_{l}-\pi\right)\right)$ for $1<$ $|z|<\exp l, \pi-\Phi_{l}<\arg z<\pi-\theta_{l}$; that $\tilde{\lambda}(z)|d z|$ satisfies (ii) of Definition 2.2 relative to the quotient metric of $\tilde{C}\left(l, \theta_{l}, \Phi_{l}\right)$ is clear. The objective is to show that $R^{*}$ is "fat" in the free homotopy class of $\gamma_{1}$ and that no new (i.e., other than $\gamma_{2}, \cdots, \gamma_{n}$ ) "pinched" free homotopy classes were introduced. Let $\gamma_{0}^{*} \subset R^{*}$ be a simple closed $\lambda_{R^{*}}$ geodesic of length less than $c^{\prime}$. If $\gamma_{0}^{*}$ does not intersect the gluing then $\gamma_{0}^{*}$ can also be considered as a curve $\gamma_{0}$ on $R$. Since $\left.\lambda_{R}\right|_{R^{*}} \leqq \lambda_{R^{*}}$ the length of $\gamma_{0}$ is also less than $c^{\prime}$. If $\gamma_{0}$ is freely homotopic to $\gamma_{1}$ then $\gamma_{0}$ can be lifted to the universal cover $H$ of $R$ with initial point $z_{0}$ and end point $z_{1}$ such that $\left|z_{0}\right|=1$ and $\left|z_{1}\right|=\exp l$. By the assumption that $\gamma_{0}^{*}$ is disjoint from the gluing the lift of $\gamma_{0}$ is disjoint from the domain $A\left(l, \Phi_{l}\right)$ and thus by estimate (2.5) has length at least $c^{\prime}$, a contradiction. By Lemma $2.7 \gamma_{0}^{*}$ cannot intersect and yet be distinct from the geodesics $\gamma_{2}, \cdots, \gamma_{n}$. Thus $\gamma_{0}$ must be freely homotopic to one of $\gamma_{2}, \cdots, \gamma_{n} \subset R$ or $\gamma_{0}^{*}$ intersects the gluing. If $\gamma_{0}^{*}$ is contained in $A\left(l, \theta_{l}, \Phi_{l}\right)$ then it must be freely homotopic to $\gamma_{1}$ a case considered above; otherwise $\gamma_{0}^{*}$ intersects the gluing and the boundaries of $A\left(l, \theta_{l}, \Phi_{l}\right)$ hence crosses the domain. By estimate (2.4) $\gamma_{0}^{*}$ has length at least $c^{\prime}$ in terms of the $\left.\lambda_{R}\right|_{R^{*}} \leqq \lambda_{R^{*}}$ metric, a contradiction. Thus $\gamma_{0}^{*}$ is freely homotopic to one of $\gamma_{2}, \cdots, \gamma_{n}$. The deformation corresponding to the replacing of $A\left(l, \theta_{l}\right)$ by $A\left(l, \theta_{l}, \Phi_{l}\right)$ can be realized in terms of quasiconformal maps. For $A=A\left(l, \theta_{l}, \Phi_{l}\right)=$ $\{z|1<| z \mid<\rho\}$ the domain $A\left(l, \theta_{l}\right)$ corresponds to the deformation of $A$ given by the element $(t-1 / t+1)(z /|z|)^{2} d z / d z \in M\left(A\left(l, \theta_{l}, \Phi_{l}\right)\right)$ where $t=\left(\pi-2 \theta_{l}\right) / 2\left(\Phi_{l}-\theta_{l}\right)$. We consider $(\tau-1 / \tau+1)(z /|z|)^{2} \overline{d z} / d z$ restricted to $A\left(l, \theta_{l}, \Phi_{l}\right) \subset R^{*} 1 \leqq \tau \leqq t$ as a curve in $M\left(R^{*}\right)$. The estimate for an annulus given by (1.4) can be now applied upon noting that $\left.\lambda_{R}\right|_{A} \leqq \lambda_{A}$ and $\left.Q(R)\right|_{A} \subset Q(A),[16]$. The Weil-Petersson length of this curve is seen to be bounded in terms of $E\left(A\left(l, \theta_{l}, \Phi_{l}\right)\right)^{1 / 2}$. Estimate (2.6) bounds the latter quantity by the constant $c_{2}^{1 / 2}$. Repeating this "fattening" process $n$ times a surface $\tilde{R} \in K_{c}$, is obtained. By Lemma $2.7 n \leqq 3 g-3$; the above remarks now yield $\omega(R, \tilde{R}) \leqq$ $(3 g-3) c_{2}^{1 / 2}$. The proof is complete.

\section{The Poincaré diameter and length of the shortest} closed geodesic. Let $R$ be a compact Riemann surface of genus $g$, $g \geqq 2$. Let $l(R)$ denote the length of the shortest closed Poincaré geodesic and $d(R)$ the Poincare diameter of $R$. The following lemma is a consequence of the considerations of 2 . 
LEMma 3.1. There exist constants $\overline{c_{1}}$ and $\overline{c_{2}}$ depending only on the genus such that

$$
\ln \left(\bar{c}_{1} / l(R)\right) \leqq d(R) \leqq 6 g \ln \left(\bar{c}_{2} / l(R)\right) .
$$

Proof. Maintaining the constants $c_{1}, c_{2}, c_{3}$ and $c^{\prime}$ of $\S 2$ we consider a surface $R \in K_{c^{\prime}}$. As $K_{c^{\prime}}$ is compact $l(R)$ and $d(R)$ are bounded above and below hence constants $\tilde{c}_{1} \tilde{c}_{2}$ exist to yield

$$
\ln \left(\tilde{c}_{1} / l(R)\right) \leqq d(R) \leqq 2 \ln \left(\tilde{c}_{2} / l(R)\right)
$$

for surfaces in $K_{c^{\prime}}$. Now let $R \notin K_{c^{\prime}}$ then clearly $d(R)$ is bounded below by one-half the width of $A\left(l, \theta_{l}\right) \subset R$ where $l=l(R)$. Thus

$$
\ln \left(c_{2} / 2 l\right) \leqq \ln \left(\cot \theta_{l}+\csc \theta_{l}\right) \leqq d(R) .
$$

Setting $\overline{c_{2}}=\min \left\{c_{2}, \tilde{c}_{2}\right\}$ the lower bound is established. Assume that $R \notin K_{c^{\prime}}$ and has only one closed Poincaré geodesic of length less than $c^{\prime}$. Forming the surface $R^{*}$ as in 2. by removing $A\left(l, \Phi_{l}\right)$ from $A\left(l, \theta_{l}\right) \subset R$ where $l=l(R)$ we have that $d(R)$ is bounded by the sum of the width of $A\left(l, \theta_{l}\right), l / 2$ and $d\left(R^{*}\right)$. Specifically for two points $x, y$ of $R^{*}$ we connect them with a $\lambda_{R}$. length minimizing curve $\gamma_{x, y}$. If this curve intersects the gluing a new curve is formed as the union of the shortest segment of $\gamma_{x, y}$ from $x$ to the gluing, a segment along the gluing and the shortest segment of $\gamma_{x, y}$ from the gluing to $y$. Now taking account of the relation of $R$ to $R^{*} d(R)$ is seen to be bounded by

$$
2 \ln \left(\tilde{c}_{2} / l(R)\right)+c^{\prime}+2 \ln \left(\tilde{c}_{2} / l\left(R^{*}\right)\right)
$$

where $\tilde{c}_{2}$ has been appropriately modified. A constant $\overline{c_{2}}$ can now be chosen to bound this last quantity by $4 \ln \left(\overline{c_{2}} / l(R)\right)$. In general let $S$ be a surface with exactly $n$ closed Poincaré geodesics of length less than $c^{\prime}$. We claim that $d(S) \leqq 2(n+1) \ln \left(\overline{c_{2}} / l(S)\right)$ for an appropriate $\overline{c_{2}}$. Proceeding by induction on $n$ it remains only to consider the induction step. Let $R \notin K_{c^{\prime}}$ have exactly $n+1$ closed Poincaré geodesics of length less than $c^{\prime}$. Forming the surface $R^{*}$ and arguing as above $d(R)$ is bounded by the sum of the width of $A\left(l, \theta_{l}\right) \subset R, l / 2$ and $d\left(R^{*}\right)$ where $l=l(R)$. Using the induction hypothesis this is bounded by

$$
2 \ln \left(\tilde{c}_{2} / l(R)\right)+c^{\prime}+2(n+1) \ln \left(\tilde{c}_{2} / l\left(R^{*}\right)\right)
$$

which in turn is bounded by

$$
2(n+2) \ln \left(\overrightarrow{c_{2}} / l(R)\right) .
$$


Observing that $n$ is at most $3 g-3$ the upper bound is now established.

In contrast to the present lemma the constructive estimate

$$
d(R) \leqq(g-1) l(R) / \sinh ^{2}(l(R) / 2)
$$

where

$$
l(R) / \sinh ^{2}(l(R) / 2) \approx 4 / l(R)
$$

for $l(R)$ sufficiently small was given by L. Bers, [4].

\section{REFERENCES}

1. L. V. Ahlfors, Some remarks on Teichmüller's space of Riemann surfaces, Ann. of Math., 74 (1961), 171-191.

2. — Conformal Invariants, McGraw-Hill, New York, N.Y., 1973.

3. L. Bers, Quasiconformal mapping and Teichmüller's theorem, Analytic Functions by R. Nevanlinna, et al., Princeton University Press, Princeton, N.J., 1960, 89-119.

4. - A remark on Mumford's compactness theorem, Israel J. Math., 12 (1972), 400-407.

5. — Uniformization, moduli, and Kleinian groups, Bull. London Math. Soc., 4 (1972), $257-300$.

6. __ Fiber spaces over Teichmüller spaces, Acta Math., 130 (1973), 89-126.

7. T. C. Chu, Noncompleteness of the Weil-Petersson metric, preprint.

8. — Thesis, Columbia University, 1976.

9. N. Halpern, Collars on Riemann surfaces, preprint.

10. L. Keen, Collars on Riemann surfaces, Ann. of Math. Studies, 79 (1974), 263-268.

11. S. Kravetz, On the geometry of Teichmüller spaces and the structure of their modular groups, Ann. Acad. Sci. Fenn., 278 (1959), 1-35.

12. H. Masur, The extension of the Weil-Petersson metric to the boundary of Teichmüller space, preprint.

13. D. Mumford, A remark on Mahler's compactness theorem, Proc. Amer. Math. Soc., 28 (1971), 289-294.

14. H. E. Rauch, A transcendental view of the space of algebraic Riemann surfaces, Bull. Amer. Math. Soc., 71 (1965), 1-39.

15. H. L. Royden, Invariant metrics on Teichmüller space, Contributions to Analysis by L. V. Ahlfors, et al., Academic Press, New York, N.Y., 1974, 393-399.

16. S. Wolpert, Non-completeness of the Weil-Petersson metric for Teichmüller space, Pacific J. Math., 61 (1975), 573-577.

17. The Weil-Petersson metric for Teichmüller space and the Jenkins-Strebel differentials, Thesis, Stanford University, 1976.

Received June 23, 1976.

UNIVERSITY OF MARYLAND

COllege Park, MD 20742 



\section{Pacific Journal of Mathematics \\ Vol. 70, No. $1 \quad$ September, 1977}

William H. Barker, Noether's theorem for plane domains with hyperelliptic

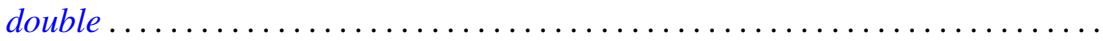

Michael James Beeson, Non-continuous dependence of surfaces of least area on the boundary curve ...................................... 11

Horst Behncke, Functions acting in weighted Orlicz algebras . . . . . . . . . . . . 19

Howard Edwin Bell, A commutativity study for periodic rings . . . . . . . . . . . 29

Peter Botta and Stephen J. Pierce, The preservers of any orthogonal group ....... 37

Douglas S. Bridges, The constructive Radon-Nikodým theorem ............. 51

James Dennis Brom, The theory of almost periodic functions in constructive

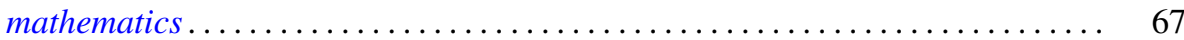

N. Burgoyne and C. Williamson, Semi-simple classes in Chevalley type groups ....

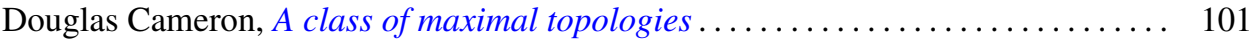

L. Carlitz, Enumeration of doubly up-down permutations . . . . . . . . . . . . . . 105

Paul Robert Chernoff, The quantum n-body problem and a theorem of

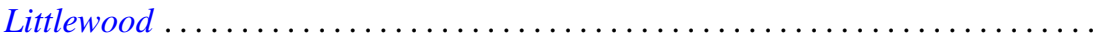

Jo-Ann Deborah Cohen, Locally bounded topologies on $F(X) \ldots \ldots \ldots \ldots \ldots \ldots$

Heinz Otto Cordes and Robert Colman McOwen, Remarks on singular elliptic

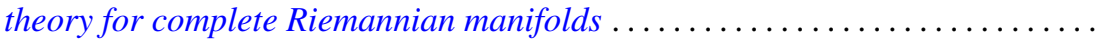

Micheal Neal Dyer, Correction to: "Rational homology and Whitehead

products"

Robert Fernholz, Factorization of Radonifying transformations

Lawrence Arthur Fialkow, A note on quasisimilarity. II ...... . .

Harvey Charles Greenwald, Lipschitz spaces of distributions on the surface of unit

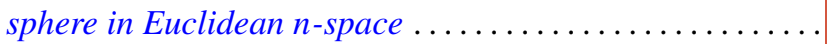

Albrecht Irle, On the measurability of conditional expectations

Tom (Roy Thomas Jr.) Jacob, Matrix transformations involving simple sequence

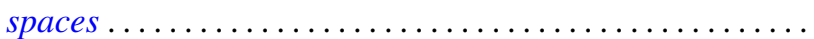

A. Katsaras, Continuous linear maps positive on increasing continuous

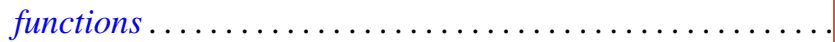

Kenneth Kunen and Judith Roitman, Attaining the spread at cardinals of cofinality

Lawrence Louis Larmore and Robert David Rigdon, Enumerating normal bundles

of immersions and embeddings of projective spaces ...... . .

Ch. G. Philos and V. A. Staïkos, Asymptotic properties of nonoscillatory solutions of differential equations with deviating argument .

Peter Michael Rosenthal and Ahmed Ramzy Sourour, On operator algebras containing cyclic Boolean algebras...

Polychronis Strantzalos, Strikt fast gleichgradig-stetige und eigentliche

Aktionen ...

Glenn Francis Webb, Exponential representation of solutions to an abstract

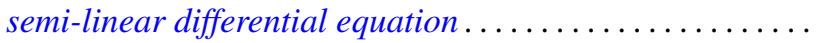

